\title{
Research on cutting tool wear based on fractional Brownian motion
}

\author{
Liang Jian-kai, Song Wan-qing, Li Qing \\ College of Electronic and Electrical Engineering, Shanghai University of Engineering Science, Shanghai, P.R. China \\ Email address: \\ liangjiankai126@126.com (Liang Jian-kai)
}

\section{To cite this article:}

Liang Jian-kai, Song Wan-qing, Li Qing. Research on Cutting Tool Wear Based on Fractional Brownian Motion. International Journal of Mechanical Engineering and Applications. Vol. 3, No. 1, 2015, pp. 1-5. doi: 10.11648/j.ijmea.20150301.11

\begin{abstract}
Cutting tool wear is a very complex process. Various factors have a direct or indirect effect on cutting tool wear, resulting in uncertainty, so it is difficult for experimental data and result to have good stability. However, Vibration analysis is a very important means for condition monitoring and fault diagnosis. This paper aims to study the methods of tool vibration signal processing, pattern recognition and trend prediction. Collected on tool vibration signal at different times, wavelet noise reduction is used to pretreat the vibration signals. Then, for the self-similar vibration signals, we propose the fractional Brownian motion (FBM) theory with long-range dependence (LRD). Combined with Wigner-Ville spectrum, characteristic parameter can be extracted, so the cutting tool wear state can be determined according to fractal dimension and average slope of the fitting curve of the logarithm power spectrum. Finally, we use FBM model to predict the trend of tool vibration signals. Experiments show that the methods have a good effect on tool wear state recognition and trend prediction.
\end{abstract}

Keywords: Tool Wear, Fractal Dimension, Wigner-Ville Spectrum, FBM Model, Trend Prediction

\section{Introduction}

In the machining process, the tool wear will affect the surface quality and dimensional accuracy of the artifacts. Therefore, monitoring and predicting tool wear more accurately are currently an urgent issue to be solved in the automatic machining process ${ }^{[1]}$. Studies have shown that tool condition monitoring and diagnosis face a lot of non-stationary signals. Researching the new practical methods are the need to promote the continuous development of tools fault diagnosis.

Bhattacharyya used cutting force signal to estimate tool wear in face milling. Stephenson and Ali performed studies on tool temperature effects on interrupted metal cutting and reported theoretical and experimental results ${ }^{[2-3]}$. Iwata and Moriwaki used an acoustic emission signal to monitor tool wear condition in cutting processes. Zhang et al. used a Hall Effect sensor to measure the current supplied to the spindle motor drive of a vertical NC miller together with the cutting forces ${ }^{[4-5]}$. According to trend prediction, prediction experts have put forward a prediction method, which adopts a particle swarm optimization extended memory method which combines support vector regression (SVR) and a prediction method which combines support vector machines (SVM) and wavelet neural network optimization ${ }^{[6-7]}$. This improves the accuracy of prediction, but has a complex computing process.

In the paper, combined FBM theory with Wigner-Ville spectrum, the cutting tool wear state can be determined according to fractal dimension and average slope of the fitting curve of the logarithm power spectrum. Then, the paper also proposes the FBM model and calculates the model parameters to predict the future tool vibration signals.

\section{The Method of Tool Wear State Recognition}

\subsection{Fractional Brownian Motion (FBM)}

If $0<\mathrm{H}<1$, the fractional Brownian motion (FBM) with Hurst parameter $\mathrm{H}$ is the continuous Gaussian process $\left\{B_{H}(t, w), t>0\right\}, B_{H}(0, w)=b_{0}$ and so whose definition is given by ${ }^{[8]}$ :

$$
\begin{aligned}
& \mathrm{B}_{\mathrm{H}}(t, w)-B_{H}(0, w) \\
& =\frac{1}{\Gamma(1+\alpha)}\left\{\int_{-\infty}^{0}\left[(t-s)^{\alpha}-(-s)^{\alpha}\right] d B(s, w)+\int_{0}^{t}(t-s)^{\alpha} d B(s, w)\right\}
\end{aligned}
$$


Where $\alpha=H-1 / 2, B(s, w)$ is a weiner process and $\Gamma(1+\alpha)=\int_{0}^{\infty} x^{\alpha} e^{-x} d x$

If $\mathrm{H}=\frac{1}{2}$ then $B_{H}(t, w)$ coincides with the standard Brownian motion $\mathrm{B}(\mathrm{t}, \mathrm{w})$.

The constant $\mathrm{H}$ determines the sign of the covariance of the future and past increments. This covariance is positive when $\mathrm{H}>\frac{1}{2}$, zero when $\mathrm{H}=\frac{1}{2}$ and negative when $\mathrm{H}<\frac{1}{2}$.

\subsection{Wigner-Ville Spectrum}

FBM is a non-stationary process. For the non-stationary process, its power spectrum does not have a clear definition, so it cannot be obtained by the usual methods. But by Wigner-Ville spectral decomposition, we get the power spectrum.

If the average power of fractional Brownian function $B_{H}(t)$ is limited, then

$$
\lim \frac{1}{2 T} \int_{-t}^{t}\left|\mathrm{~B}_{\mathrm{H}}(\mathrm{t})\right|^{2} d t<+\infty
$$

So the power spectrum $\mathrm{B}_{\mathrm{H}}(\mathrm{t})$ is proportional to $f^{-2 H-1}[9]$, then

$$
\lim \frac{1}{2 T}\left|\int_{-t}^{t} \mathrm{~B}_{\mathrm{H}}(\mathrm{t}) e^{-j 2 \pi f t} d t\right|^{2}=C f^{-2 H-1}
$$

For non-stationary random process $\mathrm{X}(\mathrm{t})$, if the correlation function $R(t, s)$, then the Wigner- Ville spectrum of $X(t){ }^{[10]}$ is

$$
W_{x}(t, w)=\int_{-\infty}^{+\infty} R_{x}\left[t+\frac{\tau}{2}, t-\frac{\tau}{2}\right] e^{-i w t} d \tau
$$

So Wigner-Ville spectrum is a time-dependent spectrum, Wigner-Ville spectrum of FBM $S_{B_{H}}(w)$ is

$$
S_{B_{H}}(w)=\lim _{T \rightarrow \infty} S_{B_{H}}(w, \mathrm{~T})=\left(1 /|w|^{2 H+1}\right)
$$

If the function $\mathrm{Z}(\mathrm{x})$ subjects to FBM of variable $\mathrm{x}$, then the power spectrum of $Z(x)$ is bound to obey the formula 4 and get the power spectrum of $Z(x)$ :

$$
S_{Z}(w)=\frac{1}{|w|^{2 H+1}}
$$

Take the logarithm to formula 6 :

$$
\log \left(S_{Z}(w)\right)=-(2 H+1) \log (w)
$$

If the slope of the line is $\mathrm{k}$, you can get the fractal parameter $\mathrm{H}=-(\mathrm{k}+1) / 2$. The relationship about fractal parameters $\mathrm{H}$ and fractal dimension $\mathrm{D}$ of FBM is: $\mathrm{D}=2-\mathrm{H}$, so we can get $\mathrm{D}=$ $(5-\mathrm{k}) / 2$.
By calculating the power spectrum of vibration signals can we plot the logarithmic plot of power spectrum, the slope of the fitted line can be obtained by the least squares fitting. By analyzing the values of the parameter $\mathrm{D}$ in different time periods, we can find the regularity and judge the state of tool wear.

\subsection{The FBM Model}

\subsubsection{FBM Incremental Simulation}

If time interval $[0, \mathrm{~T}]$ is divided into $\mathrm{N}$ equal parts, then each length is $\Delta t=T / N$.For each time interval $t_{j}$ $(j=0,1,2 \cdots, N)$, FBM increment is discretized by Maruyama extended symbols ${ }^{[11-12]}$ :

$$
\Delta B\left(\mathrm{t}_{j}, \mathrm{H}\right)=\Delta B\left(\mathrm{t}_{j}+\Delta \mathrm{t}, \mathrm{H}\right)-B\left(\mathrm{t}_{j}, \mathrm{H}\right)=w(t)(\Delta t)^{H}
$$

If $\mathrm{T}=1, \mathrm{~N}=200,=0.005, \mathrm{H}=0.65$, then we get incremental simulation diagram of FBM and see FBM with steady increments.

According to $\mathrm{B}\left(\mathrm{t}_{j+1}, H\right)=B\left(t_{j}, H\right)+w(t)(\Delta t)^{H}$, we get the FBM simulation curves, shown in Fig1.
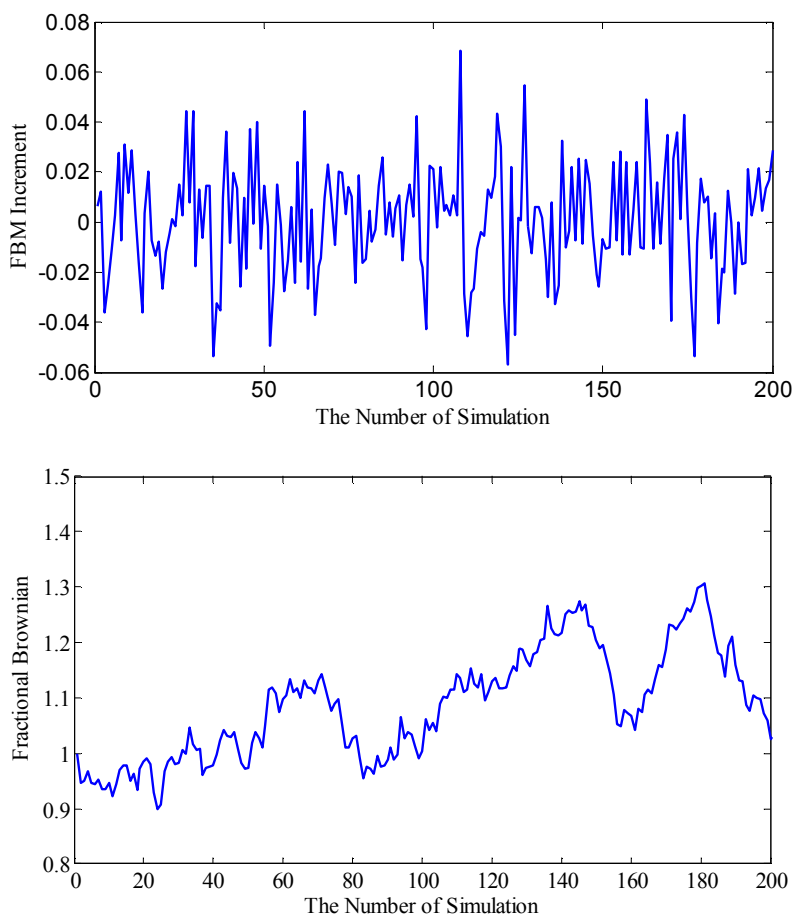

Fig. 1. FBM and its incremental simulation

\subsubsection{FBM Stochastic Simulation}

Simulate the vibration signals and its changes meet the following fractional stochastic differential equation.

$$
d \mathrm{~S}=\mu S d t+\sigma S d B_{H}+\lambda S\left(d B_{H}\right)^{2}
$$

Where $\mu$ is the drift rate, $\sigma$ is the fluctuation rate of gain, $\lambda$ is the interference term of gain.

The essential difference between this model and standard 
Brown motion-driven model is that fractional Brown motion can be described the long-term memory of returns, and the increment is not independent of each other.

In this paper, the expansion of Maruyama symbol $\mathrm{d} B_{H}=w(t)(d t)^{H}$ is used to simulate the FBM increment, the time period is divided into $M$ equal-spaced intervals, the time interval is $\Delta \mathrm{t}$, discrete stochastic differential equation ${ }^{[13]}$ :

$$
\begin{aligned}
\mathrm{S}_{t+1} & =\mathrm{S}_{t}+\mu S_{t} \Delta t+\sigma S_{t} w_{1}(t)(\Delta t)^{H} \\
& +\lambda S_{t} w_{2}(t)(\Delta t)^{2 H}
\end{aligned}
$$

Where $w_{1}(t)$ and $w_{2}(t)$ are independent which are standard normal distributions.

Take the initial signal $\mathrm{S}_{0}, \mathrm{FBM}$ simulation of the time period is shown in Fig2.

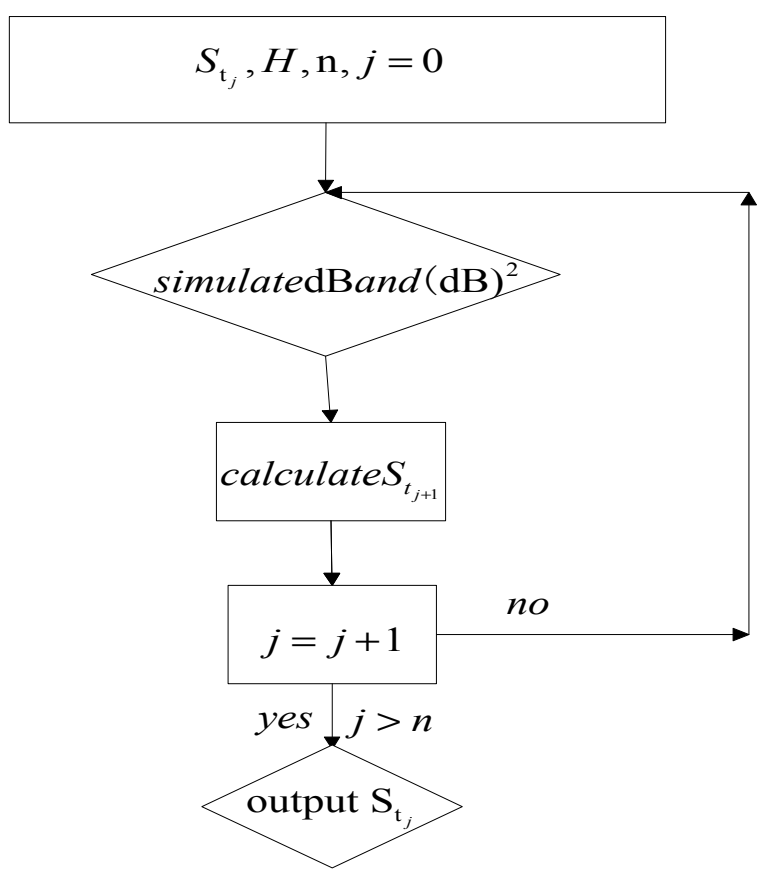

Fig. 2. Flow chart of signal simulation

\subsubsection{Parameters Estimation}

Assuming time interval of the gain data is $\Delta \mathrm{t}$, the $\mathrm{N}+1$ data of observation vector consist of $\mathrm{y}=\left(y_{0}, y_{\Delta t}, \cdots y_{N \Delta t}\right)$, time vector is $\mathrm{t}=(0, \Delta t, \cdots, N \Delta t)$, fractional Brown motion vector is $B_{H}(t)=\left[B_{H}(0), B_{H}(\Delta t), \cdots, B_{H}(N \Delta t)\right]$, so the parameters $\mu$ and $\sigma$ by the maximum likelihood estimation is as follows ${ }^{[14]}$ :

$$
\begin{gathered}
\tilde{u}=\frac{t \Gamma_{H}{ }^{-1} y^{\prime}}{t \Gamma_{H}{ }^{-1} t^{\prime}} \\
\tilde{\sigma}^{2}=\frac{1}{N} \frac{\left(y \Gamma_{H} y^{\prime}\right)\left(t \Gamma_{H} t^{\prime}\right)-\left(t \Gamma_{H} y^{\prime}\right)^{2}}{t \Gamma_{H}{ }^{-1} t^{\prime}}
\end{gathered}
$$

Parameter $\lambda$ can be obtained by fourth-order matrix describing the extreme phenomenon:

$$
\tilde{\lambda}=\sqrt[4]{E(\mathrm{y}-E(y))^{4}}
$$

Where

$$
\begin{gathered}
y=\frac{S_{t+1}-S_{t}}{S_{t}}(t=0,1,2 \cdots, N) \\
\Gamma_{H}=\left[\operatorname{cov}\left[B_{H}(i \Delta t), B_{H}(j \Delta t)\right]\right]_{i, j=0,1,2 \cdots, N} \\
=\frac{1}{2}(\Delta t)^{2 \mathrm{H}} \cdot\left(\mathrm{i}^{2 H}+j^{2 H}-|i-j|^{2 H}\right)_{i, j=0,1,2 \cdots, N}
\end{gathered}
$$

In this paper, $\mathrm{R} / \mathrm{S}$ method is calculated to Hurst parameter $\mathrm{H}$ of input sequence. Hurst coefficient $H \in(0,1)$ is a measure of a persistent random phenomenon. $H \in(0.5,1)$ can have a long- range dependence. The greater the value $H$ is, the stronger the long- range dependence is. Hurst parameter on signal series is 0.6497 , as shown in Fig3.

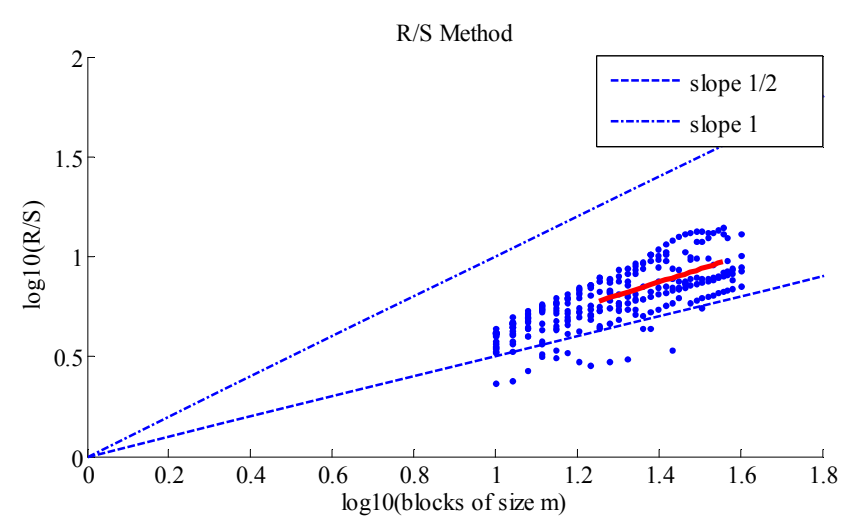

Fig. 3. Hurst index of vibration signals

According to the selected data as a calculation cycle, the parameters result is

$$
\mu=0.6011, \quad \sigma^{2}=0.0013, \lambda=0.0281
$$

Through multiple simulations, we can get number of vibration signals approximate curves, then average for each time point of all possible approximation load curves, you can get on the approximate vibration signals values of each time point, that is the most likely path changes.

\section{The Experiment Simulation}

\subsection{Tool Wear State Recognition}

In this paper, we adopt $\mathrm{db}$ wavelets to denoise signals. Due to use different $\mathrm{db}$ wavelets, we can get different effects. And $\mathrm{db} 4$ wavelet relative to other wavelets has the shortest time window, and better time resolution, so we use $\mathrm{db} 4$ wavelet to denoise signals ${ }^{[15]}$. Because the selection and quantification of threshold is directly related to the quality of signal denoising, the paper selects the default threshold to denoise signals. 
Collected 4096 tool vibration signals data for a period of time, it was divided four sections for analysis, the

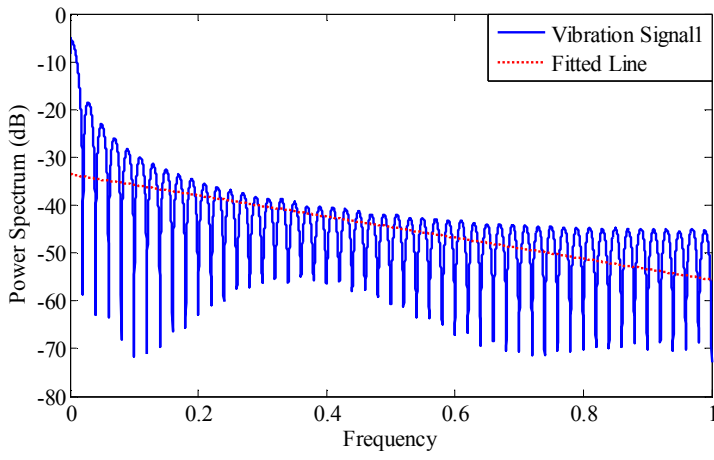

(a)The power spectrum of vibration signal1

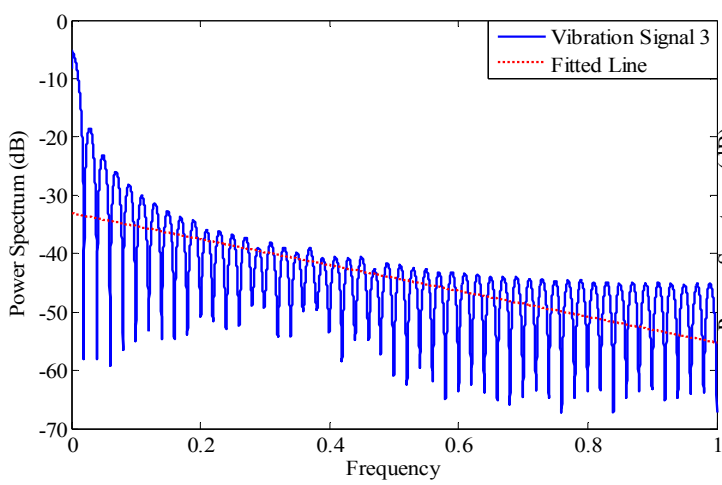

(c)The power spectrum of vibration signal3 corresponding logarithmic power spectrum and fitted curve are shown in Fig4.

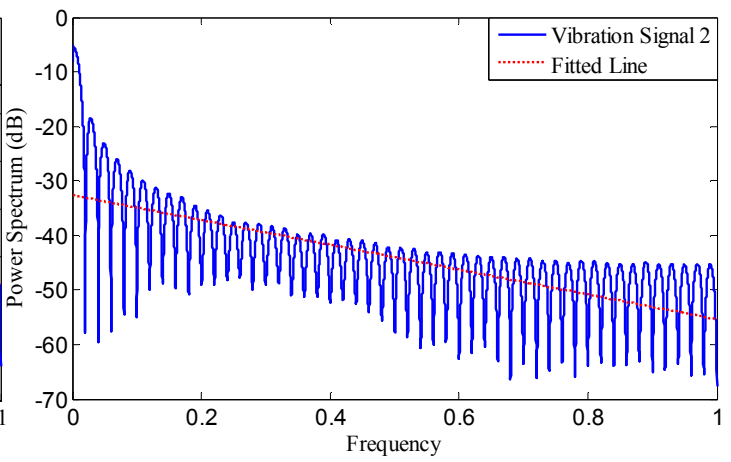

(b)The power spectrum of vibration signal2

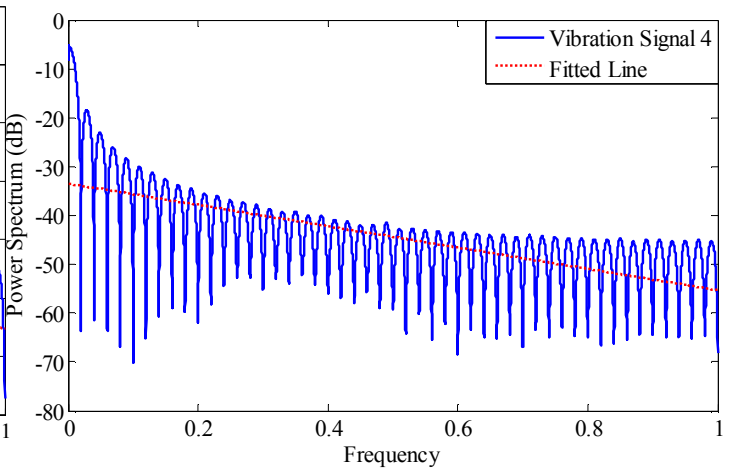

(d)The power spectrum of vibration signal4

Fig. 4. The power spectrum and fitted curve of vibration signals

According to the Fig4, the average slope of the time period and fractal dimension $\mathrm{D}$ can be calculated by the fitting slopes, and we can in turn to calculate the fractal dimension $\mathrm{D}$ of other periods.
As can be seen from the Tab1, with the increase of the tool cutting time, the fractal dimension D of the Brownian motion of the vibration signal is also increasing. Thus, the larger fractal dimension $\mathrm{D}$, the greater the tool wear.

Table 1. The fractal dimension parameters

\begin{tabular}{llllll}
\hline Time Parameter & 0 minute & 20 minute & 40 minute & 60 minute \\
\hline $\mathrm{D}$ & 13.54 & 13.69 & 14.44 & 14.96 & 15.7 \\
\hline
\end{tabular}

\subsection{Trend Forecast}

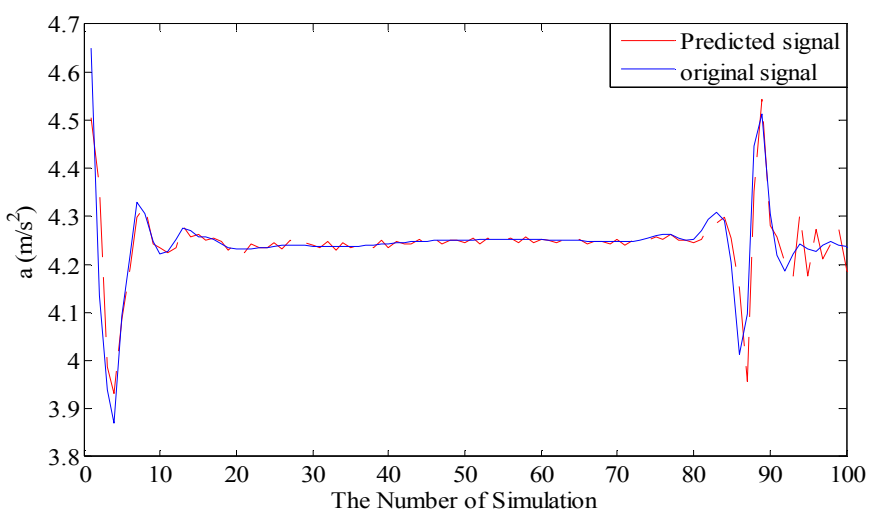

Fig. 5. Original and predicted values of vibration signals

According to the FBM model, we collect the tool vibration signals at a certain time and predict the future situation of vibration signals ${ }^{[16]}$. The next 100 vibration signals data are simulated and compared with the real values by using MATLAB, as it is shown in Fig5.

Figure 5 can clearly be seen that FBM model can relatively accurate predict the future values, the maximum relative error of FBM model is within $6 \%$. From the terms of the maximum and minimum relative error, or the average relative error, the method has slightly satisfactory prediction results and provide a new method for predicting tool state.

\section{Conclusions}

For the characteristics of vibration signals of the tool wear state, it is studied on the application of FBM and Wigner-Ville methods to the tool wear state identification, three main conclusions are as follows:

- In terms of the instability, nonlinearity and long-range 
dependence of vibration signals, the paper proposes the wavelet theory and FBM method combined with Wigner-Ville Spectrum;

- FBM can reflect the long-range dependence advantages of time series, combined FBM theory with Wigner-Ville spectrum, the cutting tool wear state can be determined according to fractal dimension and average slope of the fitting curve of the logarithm power spectrum;

- FBM model used in economy and network traffic is proposed to establish stochastic differential equation and simulation has a better prediction result and provides an effective method to study the long-term trend prediction.

\section{Acknowledgements}

This project is supported by Shanghai Natural Science Foundation of China (Grant No.14ZR1418500). The authors would like grateful to the editors and reviewers for their constructive comments.

\section{References}

[1] D.E.Dimla, P.M.Lister, On-line metal cutting tool condition monitoring: force and vibration analyses, Int. J. Mach. Tools Manuf., 5(40), pp. 739-768, 2000.

[2] P. Bhattacharyya, D. Sengupta, S. Mukhopadhyay, Cutting force-based real-time estimation of tool wear in face milling using a combination of signal processing techniques. Mechanical Systems and Signal Processing, 6(20), pp. 2665-2683, 2007.

[3] D. A. Stephenson, A. Ali, Tool temperatures in interrupted metal cutting, Winter Annual Meeting of the ASME, pp. 261-281, 1990.

[4] K.Iwata, T. Moriwaki, Application of acoustic emission measurement to in-process sensing of tool wear, Ann. CIRP 26 (1-2), pp. 19-23, 1977.

[5] D. Zhang, S. Dai, Y. Han, D. Chen, On-line monitoring of tool breakage using spindle current in milling, In:1st Asia-Pacific and 2nd Japan-China International Conference Progress of Cutting and Grinding, Shanghai, China, pp. 270-276, 1994.

[6] Hong W C, Chaotic particle swarm optimization algorithm in a support vector regression electric load forecasting model, Energy Conversion and Management, 50(1), pp.105-117, 2009.

[7] Zhang Q, Lai K K, Niu D, Optimization Combination Forecast Method of SVM and WNN for Power Load Forecasting, IEEE Transactions on Computational Sciences and Optimization (CSO), pp.249-253, 2011.

[8] Lundahl T, Ohley W J, Kay S M, et al, Fractional Brownian motion: A maximum likelihood estimator and its application to image texture, IEEE Transactions on Medical Imaging, 5(3), pp.152-161, 1986.

[9] Jeon, Jae-Hyung, Fractional Brownian motion and motion governed by the fractional Langevin equation in confined geometries, Physical review, 2(81), 2010.

[10] S. Ghofrani, D.C. McLernon, Auto-Wigner-Ville distribution via non-adaptive and adaptive signal decomposition, Signal Processing, 8(89), pp.1540-1549, 2009.

[11] Longjin Lv, Ren Fu-Yao, The application of fractional derivatives in stochastic models driven by fractional Brownian motion, Physical a-statistical mechanics and its applications, 21(389), pp.4809-4818, 2010.

[12] Didier, Gustavo, Pipiras, Vladas, Integral representations and properties of operator fractional Brownian motions, Bernoulli, 1(17), pp.1-33, 2011.

[13] Wang li-li, Numerical Calculation and Empirical Analysis of American Options Pricing Based On Fractional Brownian Motion, Huazhong University of science and technology, 2012.

[14] Xiao we-lin, Research on the pricing method for warrants of long memory processes. South China University of science and technology, 2010.

[15] M. Kious, A. Ouahabi, Detection process approach of tool wear in high speed milling, Measurement, 10(43), pp.1439-1446, 2010 .

[16] H. Saglam, A. Unuvar, Tool condition monitoring in milling on cutting forces by a neural network, Int. J. Prod. Res., 41 (7), pp. 1519-1532, 2003. 